\title{
Quality-of-Information Aware Transmission Policies with Time-Varying Links
}

\author{
Ertugrul Necdet Ciftcioglu and Aylin Yener \\ Dept. of Electrical Engineering, The Pennsylvania State University, University Park, PA 16802 \\ Email:enc118@psu.edu, yener@ee.psu.edu
}

\begin{abstract}
We consider Quality-of-Information (QoI) aware transmission policies in the presence of time-varying links in a mobile ad hoc network. QoI, tailored for military tactical networks, is defined by a set of attributes relevant to the application. Time-varying nature of links in practical networks leads to uncertainty in evaluating QoI utility to be delivered to end users. This delivered-QoI utility is a function of both attributes provided by the source input, as a result of observing certain events, and the channel induced attributes that impact the QoI obtained at the destination. The goal of this paper is to attain the maximum QoI output utility, termed as Operational Information Content Capacity (OICC) of a network. First, for a single link, we demonstrate that the optimal decision structure for transmission is threshold-based. Next, we consider multihop relay networks. For the basic model of a two-hop relay network, we propose transmission scheduling and link activation schemes based on approximate dynamic programming methods. Furthermore, we exploit time-variations of links by opportunistic scheduling by employing buffers at the relay node. We demonstrate that significant gains in QoI output utility are gained by opportunistic scheduling algorithms.
\end{abstract}

\section{INTRODUCTION}

In tactical networks, the main goal is sound decision making. This leads to the necessity of a new paradigm which emphasizes the quality of information by viewing the network as an information source, and developing methods to satisfy quality requirements at the end user. For military applications, Quality of Service (QoS)-based approaches that are agnostic to the application or content of data may not be sufficient. Consequently, there is growing interest in moving from traditional QoS metrics as throughput, packet delivery ratio, fairness, and delay, towards new notions of quality associated with information. Recently, a set of attributes, including provenance [1], accuracy and precision [2] [1], reliability [2], corroboration [1] [3], age/freshness and timeliness [2] [1] started to emerge as factors impacting the Quality-of-Information (QoI) [2] [1]. Initial work to assess the impact of this new paradigm on fundamental networking operations has considered the probability of error in event detection problems as a QoI metric [4].

Recently, we have proposed QoI-aware scheduling policies where new information arrives randomly to the source for a single link, trading the attributes of accuracy and freshness [5]. We have also characterized the set of utility-maximizing QoI

Research was sponsored by the U.S. Army Research Laboratory under the Network Science Collaborative Technology Alliance, Agreement Number W911NF-09-2-0053. vectors and associated rate allocation for multiuser networks [6]. All this previous work assumes a static environment, and does not address problems that might arise nor take advantage of the time varying nature of wireless channels.

We consider a tactical network where an end user requests missions to be performed sequentially, and other users with sensing capabilities respond to each mission. The utility derived at the end user depends on the QoI of the response. We are interested in the maximum utility a network can provide relevant to the desired operation, which has been termed the Operational Information Content Capacity (OICC) of the network [1]. In this paper, we consider a dynamic tactical network where channel states are time-varying. In particular, we consider the following two basic models: a single link and a two-hop relay network, with the objective of maximizing the utility of the system, i.e., attaining the OICC. The dynamic nature of channel states leads to a sequential decision making problem in order to maximize QoI utility.

Among attributes that can effect QoI, we focus particularly on accuracy and timeliness. These two attributes are fundamental representatives in the sense that accuracy is an indicator of the quality of the initial information content and generating information at the sources, while timeliness is concerned with the capability and the quality of the network to deliver the information. In Section III, we discuss a model for output QoI utility that depends on these two metrics.

The input information content can simply be characterized as an amount of data to be delivered. In the most general sense, the specific size of data in terms of bits depending on attributes including type, accuracy, precision and credibility. Depending on network delivery, a delay will be introduced until the information is utilized at the destination. This delay will depend on rates allocated to links, the number of hops in the path and possibly queueing delays.

In [7], we have demonstrated that deploying queues at relay nodes significantly improves stability regions for stochastic traffic and time-varying channels. The underlying reason was that queueing at the relay(s) allows for opportunistically exploiting the channel variations. In this paper, we shall utilize the idea of opportunistically exploiting the channel variations for QoI-based multihop relay networks.

The main contributions of this paper are twofold: (i) For a single link, we establish the optimal transmission structure to achieve the OICC. (ii) For a relay network, we propose a practical joint transmission scheduling and link activation 


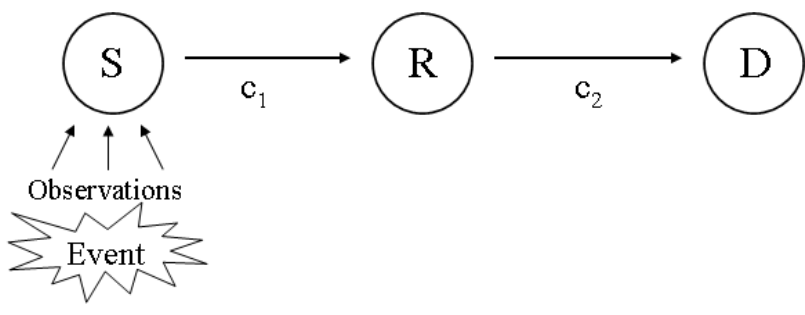

Fig. 1. Two-hop QoI-based relay network.

scheme.

\section{System Model}

For clarity of exposition, we concentrate on two fundamental communication models: a single link, and a two-hop relay network (Fig. 1). The results for the two-hop case can be readily generalized to more than two hops. These constitute basic and inspiring models for QoI adaptation, which involve scheduling among links and QoI optimization accordingly.

We consider a scenario where missions are issued from an end user in a tactical network. Missions arrive sequentially at time instants $\left\{b_{1}, b_{2}, \ldots\right\}$ with stochastic interarrival times, which are greater than or equal to $T$, i.e. $b_{s+1}-b_{s} \geq T, \forall s>$ 0 . We assume that at most one mission is processed by the network at any time. Information source $S$ responds to the mission and generates information related with the mission. This can correspond to phenomena related with the environment, as the presence of troops. The information content available at the source is associated with several attributes, as accuracy, credibility, precision, freshness which can be prioritized depending on the specific mission.

The overall importance of the information to the mission as the is characterized by the QoI of the piece of information. Two types of QoI can be defined: delivered-QoI, which is the QoI associated with a piece of information generated and delivered by the network, and desired-QoI, which is the QoI requested of the network. QoI can be represented by a QoI-vector, which is a vector of attribute-value pairs: e.g., $[$ type $=$ image, timeliness $=15 s$, accuracy $=600 \times$ $800, F O V=150 \mathrm{~mm}$ per meter ...], where FOV is the field of view which represents the (angular or linear or areal) extent of the observable world seen at any given moment. In this example, the linear FOV is given with specified in a ratio of lengths. The first term of the accuracy attribute specifies the resolution [1].

The utility derived by the end user from this response depends on its delivered-QoI. This QoI utility is a function of both the initial quality at the source and the timeliness of the information. The maximum QoI utility that the network can deliver to the end node is termed as the OICC of the network. As a result of network delivery, a delay will be introduced until the information is utilized at the destination. We are interested in the effect of delay due to network delivery on the timeliness of the information at the destination.

Once the decision of transmission is made by the source (or relay), the information available is fed into a wireless channel with a certain rate. Transmission rates can be upper bounded by the capacity of a Gaussian link given by [8]:

$$
r_{i}(t) \leq W \log _{2}\left(1+\frac{h_{i}(t) P}{N_{0} W}\right)=c_{i}(t), \quad i=1,2
$$

where $r_{i}(t)$ is the rate supported by hop $i$ at time slot $t, \sqrt{h_{i}(t)}$ denotes the channel gain for link $i$ at time slot $t, P$ is the power constraint for all nodes, the $\frac{N_{0}}{2}$ is the noise spectral density and $2 W$ is the two-sided bandwidth. We assume that nodes have half-duplex constraints, i.e., no node can transmit and receive simultaneously. Channels are time-varying within missions. More specifically, we consider the quasi-static channel model where the channels potentially change after a time duration, referred to as a slot. We assume that the time scales of interest due to timeliness requirements are large enough, along with a large operational bandwidth, allowing usage of possibly multiple codewords with sufficiently large block lengths to approach the bound in (1) during any time slot. For the twohop relay network, the relay node forwards the data received from the source node using decode and forward relaying [9]. No direct link exists between the source and destination, so assistance from the relay is mandatory. Transfer of information to the destination entitles two phases of transmission: Phase I (from source to relay), and Phase II (from relay to destination). Next, we present details two different transmission policies for the relay network.

\section{A. Transmission Policies}

1) Hop-by-hop Scheduling: In Hop-by-Hop Scheduling (HHS), relay nodes possess buffers and in each slot only one of the two transmission phases is scheduled for transmission [7]. That is, either the source node transmits to the relay (a phase I transmission) or the relay forward previously received information to the destination (a phase II transmission) in a time slot. Due to the relay queues, it is not necessary to forward information in consecutive slots of phase I and phase II. the network control policy performs the scheduling decision on which nodes will be active that slot operation. At each slot, the relay queue $x(t)$ evolves according to the following dynamics:

$$
x(t+1)=\max \left(x(t)-r_{2}(t), 0\right)+r_{1}(t) .
$$

The queue size $x(t)$ limits the amount of information that can be transferred at the second hop due to the causality constraint.

2) Immediate Forwarding: In Immediate Forwarding (IF), the relay node does not possess queues and the time slot is divided into two consecutive phases. Relay immediately forwards the traffic received in the first phase to the end nodes [10], [11]. This transmission scheme is characterized by endto-end rate regions every time-slot, given by:

$$
\mathcal{C}_{e-e}(\Delta)(t)=\min \left(\Delta(t) c_{1}(t),(1-\Delta(t)) c_{2}(t)\right),
$$

where $\Delta(t) \in[0,1]$ is a time-sharing variable representing the fraction of a time-slot that is allocated to phase I. 


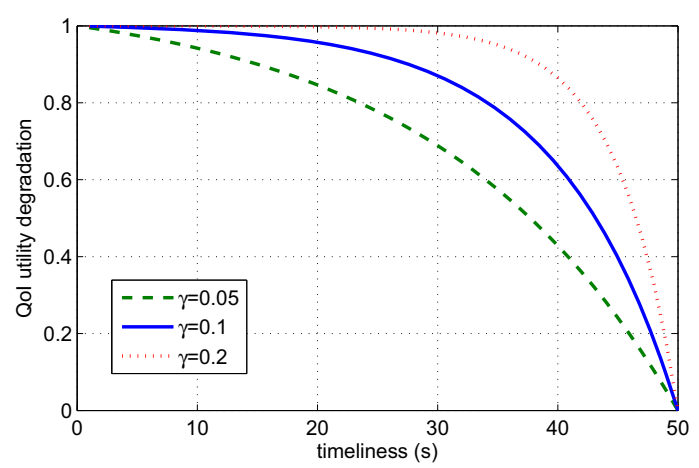

Fig. 2. Utility degradation as a function of delivery time, $D=50$.

\section{B. Utility Function Properties}

Next, we discuss a utility function which reflects the tradeoff between the attributes of accuracy and timeliness for a QoI-vector q. Consider the utility function of the following form for QoI-vector $\mathbf{q}$ :

$$
u(\mathbf{q})=a g\left(t_{d}\right),
$$

where $t_{d}$ is the timeliness, i.e. delivery time of $\mathbf{q}$, and $a$ is a scalar capturing the overall instantaneous accuracy metric of the resolution and FOV of the information specified by $q$. $g\left(t_{d}\right)$ represents the reduction in utility due to latency.

The essential properties a utility function has to satisfy with respect to timeliness is that it is non-increasing function, and it eventually decays to zero for high latency. Specifically, we focus on smooth functions which are twice differentiable and concave within the domain of interest as in [6]. As a candidate utility function, let us consider:

$$
g\left(t_{d}\right)=k(\gamma, D)\left(1-e^{\gamma\left(t_{d}-D\right)}\right),
$$

for $t_{d} \leq D$, and 0 otherwise. Illustration of sample utility degradation curves reflecting the effect of timeliness are in Fig. 2 for different parameters. The general trend observed is that the utility initially stays relatively unchanged for low delivery time and decays to zero as the delivery time approaches $D$. $D \leq T$ can be thought as a expiration time in the sense that the information is regarded useless afterwards. Application specific behavior for the utility curve can be realized by varying $\gamma \cdot k(\gamma, D)=\frac{1}{1-e^{-\gamma D}}$ is a parameter normalizing the decay.

We assume that the structure of the utility functions and behavior according to timeliness parameters are known at both of the sources, as well as channel gains.

We consider slotted operation where network decisions may be dynamically adapted at the beginning of each time slot. We also assume that the missions are all independent of each other.

For mission $s, u_{s}(t)$ denotes the amount of utility that the end user attains if the network finishes sending back its response in the current slot $t$. To facilitate online QoI-vector adaptation, we allow the option of stopping of information transfer for the mission. At the stopping instant, the end node utilizes the received information since the beginning of the mission. The overall delivered-QoI is characterized by both the accuracy through the content delivered and the timeliness through delivery time, which is in turn equivalent to the stopping time. Once the network is finished with sending information, the destination can fuse the received information at different slots and evaluate the information content which corresponds to an accuracy level and timeliness status.

More specifically, let $j$ denote the stage, i.e. number of time slots since the beginning of the ongoing current mission $s$ at any time $t$. Note that $j$ is updated as $t-b_{s}$ at each time slot $t$. At each stage $j$, the current QoI-vector $\mathbf{q}_{\mathbf{s}}(j)$ is defined by the accuracy $a_{s}(j)$ due to information already sent in previous stages of the mission, and $j$ corresponds to the timeliness. Rate regions supported vary each stage depending on $h_{1}(t)$ and $h_{2}(t)$ through (1). The increase in accuracy metric due to new information transferred at the slot, $e_{s}(j)$, depends on rate region at stage $j$. Additionally, depending on $j$, the QoIutility function experiences a degradation $g(j)$ due to latency. At stage $j, 1 \leq j<D$, the users can decide to stop, resulting in the final response with utility $u_{s}(j)$.

We define $d(j)$ : one-slot degradation factor at slot $j$, which is equal to $d(j)=\frac{g(j+1)}{g(j)}$. Then, the QoI-utility evolves according to the following dynamics:

$$
u_{s}(j+1)=d(j) u_{s}(j)+g(j+1) e_{s}(j) .
$$

$e_{s}(j)$ is equal to $e_{s, 1}(j)$ for the single link and $e_{s, 2}(j)$ for the two-hop relay network, where $e_{s, i}(j), i=1,2$, depends on $r_{i}(t)$ since transferring more bits can lead to a greater improvement on accuracy. We also note that $e_{s, 2}(j)$ is additionally limited by $x(t)$, the relay queue size.

Ultimately, the aim is to stop with maximum QoI-utility $u_{s}(j)$ with the aim of attaining QoI-vectors attaining the OICC. Note that the main obstacle is the uncertainty in future channel states and feasible rates, leading to unknown QoIutility evolution for future states.

\section{QOI OptIMIZATION}

We consider dynamically allocating resource and defining the QoI-vector. From (6), it can be readily seen that the effect of waiting for an additional slot has two opposing effects on the QoI utility. The main question of interest is to determine if the improvement in accuracy will prevail over the overall degradation due to increased delivery time, i.e., timeless. Recall that $u_{s}$ denotes the final QoI utility provided to the end user for mission $s, s \in\{1,2,3, \ldots\}$. The objective is to maximize the time average QoI utility per mission, in order to attain the OICC of the network as follows:

$$
\text { Maximize: } \lim _{H \rightarrow \infty} \frac{1}{H} \sum_{s=1}^{H} \mathbb{E}\left\{u_{s}\right\}
$$

Note that it is equivalent to maximize each mission separately to achieve the maximum in (7) since missions are independent from each other and $D \leq T$.

\section{A. Single Link}

Since separately maximizing QoI utility any mission is equivalent to maximizing the long-term average of QoI utility, 
we focus on a single mission and drop the mission subscript $s$. Also, for clarity for exposition we focus on the special case of the first mission with $b_{1}=0$, i.e. $j=t$. Then, $(u, j)$ represents the current state of the system where $u$ denotes the QoI utility state delivered to the destination and $j$ denotes the number of slots that have elapsed since the mission arrival. Let $V(u, j)$ represent the optimal value functions for state $(u, j)$. Then, $\forall j \geq 0$, Bellman's equation for this system can be written as [12]:

$$
V(u, j)=\max [u, \mathbb{E}\{V(d(j) u+g(j+1) e(t), j+1)\}]
$$

The first term within the max operator in (8) represents the utility if a stopping decision is taken at state $(u, j)$. On the other hand, the second term represents the expected utility to be attained in the case of continuing. In the event of continuing, the next state is given by $(d(j) u+g(j+1) e(t), j+1)$ with $g(j) e(t)$ representing the random increase in QoI utility due to newly delivered information at the current stage. Note that we could have equivalently written $e(j)$ instead of $e(t)$ for any mission since it only depends on the channel state at time $t$. In essence, the optimal value function at $(u, j)$ is given by the maximum of these two terms, which implies that to stop is the optimal decision at stage $j$ with utility $u$ when:

$$
u \geq \mathbb{E}\{V(d(j) u+g(j+1) e(t), j+1)\} .
$$

Otherwise, the optimal decision is to continue with the information delivery, and the extra accuracy improvement is expected to prevail over the extra time elapsing.

Recall that $D$ is the expiration time in the sense that information is useless afterwards. Each mission hence has a practical deadline of $D$ slots regardless of the next mission arrival, and we have:

$$
\begin{aligned}
& V(u, D)=0, \\
& V(u, D-1)=u, \\
& V(u, j)=\max [u, \mathbb{E}\{V(d(j) u+g(j+1) e(t), j+1)\}],
\end{aligned}
$$

with $0 \leq j \leq D-2$. Thus, it is optimal to stop at stage $D-2$ $(u, D-2)$ if $u \geq \frac{\bar{e} g(D-1)}{1-d(D-2)}$. On the other hand, for stages before $D-2$, stopping is optimal when the utility exceeds $\mathbb{E}\{V(d(j) u+g(j+1) e(t), j+1)\}$. We next demonstrate the OICC-achieving policy involves comparing utilities with stage dependent thresholds $\tau_{j}$.

Theorem 1: The OICC achieving optimal control policy is of threshold-based with stage dependent thresholds $\tau_{j}$ : At any stage $j$, stopping is optimal if the utility $u \geq \tau_{j}$, and if not the optimal decision is to continue. Furthermore, these thresholds decrease with stage $j$, i.e. $\tau_{j}>\tau_{j+1}, \forall j$.

Proof: Focusing on (9), the aim is to demonstrate that for each utility value $u$, there exists only one point of intersection with $\mathbb{E}\{V(d(j) u+g(j+1) e(t), j+1)\}$ at stage $j$. Using induction, it can be shown that the value function $V(u, j)$ is convex and increasing in utility $u$ for all stages $j$. Additionally,

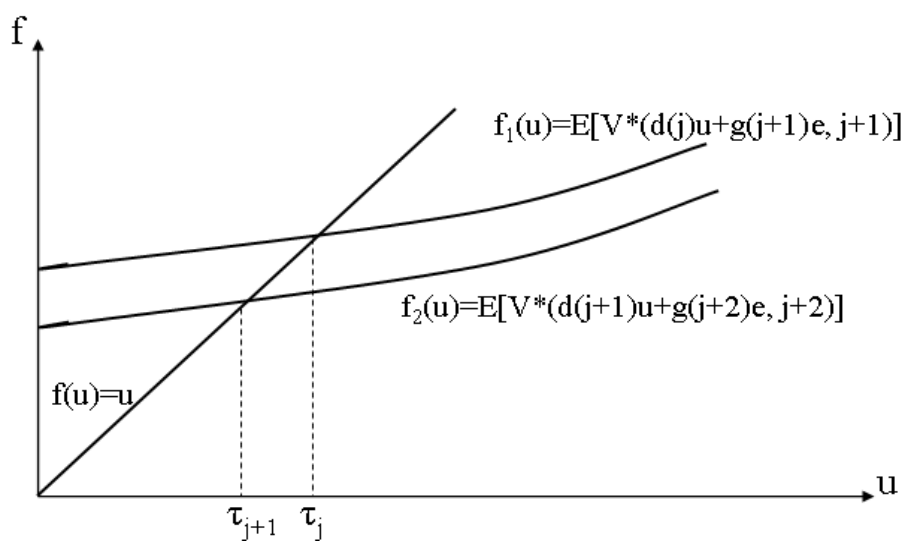

Fig. 3. Decreasing Stage-dependent thresholds.

the maximum slope of $V(u, j)$ is 1 .

Due to the fact that $V(u, j)$ has a maximum slope of 1 , it follows that the maximum slope of the function $f(u)=$ $\mathbb{E}\{V(d(j) u+g(j+1) e(t), j+1)\}$ is given by $d(j)<1$. Along with the fact that $V(0, j)>0, f(u)=u$ and $f_{1}(u)=\mathbb{E}\{V(d(j) u+g(j+1) e(t), j+1)\}$ intersect at only one point (Fig.3), given by $\tau_{j}$. Hence, the first statement of the proof is verified. For all $u$ exceeding $\tau_{j}$, the optimal decision is always to finish transmitting. Instead, $\forall u$ less than $\tau_{j}$, the optimal decision is to continue sending extra information. In other words, comparing the utility with a threshold is optimal.

Next, let us consider the second statement regarding the relation between consecutive thresholds. First, we utilize the following result:

\section{Lemma 1: $V(u, j) \geq V(u, j+1) \quad \forall u, j$}

Proof: It is possible to achieve at least the same utility as in $(u, j+1)$, i.e. $V(u, j+1)$ by the same decisions with the same utility present at the previous stage, i.e., from $(u, j)$. Combining Lemma 1 with the fact that $g(j+1)>g(j+2)$ and $d(j)>d(j+1)$ from the degradation function properties, we have that $\mathbb{E}\{V(d(j) u+g(j+1) e(t), j+1)\}>$ $\mathbb{E}\{V(d(j+1) u+g(j+2) e(t+1), j+2)\}$. As a result, the crossover point between $f(u)=u$ and $f_{1}(u)=$ $\mathbb{E}\{V(d(j) u+g(j+1) e(t), j+1)\}$, i.e., $\tau_{j}$ strictly exceeds the crossover point $\tau_{j+1}$ of $f(u)=u$ and $f_{2}(u)=$ $\mathbb{E}\{V(d(j+1) u+g(j+2) e(t+1), j+2)\} \quad$ (Fig. 3). This equivalently implies that $\tau_{j}>\tau_{j+1}$.

We note that we have observed threshold-type behavior for the different scenario and model in [5] as well, with focus on network layer issues with no emphasis on physical layer properties.

\section{Scheduling For Two-hop Relay Network}

Even though we have demonstrated that the optimal policy is threshold-based for the single link case, in practical systems solving for the dynamic program is not tractable. Moreover, it is well known that dynamic programming suffers from the curse of dimensionality when the state dimension increases. This is a drawback particularly for multihop scenarios, since queue states should also be accounted for in the decision making process as well in addition to QoI utility states. 
Hence, we resort to approximate solutions. In particular, we follow the following adaptation of the one-time lookahead approach [13], which estimate rewards from future states, to our problem at each stage $j$ :

- Compare current QoI-utility value with expected values from future decision trajectories.

- If any future trajectory offers greater expected QoI-utility than current QoI-utility, continue.

These future decision trajectories include all combinations of stopping, link scheduling and rate allocation policies, and the expectations are conditioned on the current QoI attribute states and utilities.

\section{A. Single Link}

The only decision is whether to perform stopping or not.

\section{B. Two-Hop Relay Network}

In the two-hop relay network, with immediate forwarding, it can be readily shown that the optimal time division parameter between phases is given by:

$$
\Delta(t)=\frac{c_{2}(t)}{c_{1}(t)+c_{2}(t)},
$$

leading to an end-to-end rate of $\frac{c_{1}(t) c_{2}(t)}{c_{1}(t)+c_{2}(t)}$. The immediate forwarding structure is equivalent to the single hop case, with the rate replaced by this maximum flow value each slot. Hence the only decision is again whether to stop or continue.

On the other hand, with hop-by-hop scheduling and the presence of the relay queue, there are two decisions to perform: whether to stop or not, and if the decision is to continue, which link to schedule. First, simply scheduling the second hop to deliver information at the receiver whenever the relay queue is non-empty is not an efficient way to utilize system resources due to causality requirements. Note that link scheduling has been widely studied in the opportunistic scheduling community. In the case where the objective is to maximize longterm throughput, it is well-known that max-weight algorithms are sufficient [14]. Yet, our problem is involved with much shorter time scales. Moreover, the source typically possess significantly more information compared with the relay, which would lead to strict prioritization at the first hop, which might be intolerable for many applications. Strictly prioritizing best links can lead to unbalanced resource allocation, and can reduce overall net flow to the destination. In order to strike a balance for link activation, we resort to a modified version of the proportional fair queueing (PFQ) scheduling algorithm [15]. Each slot $t$, if the decision is to continue, the controller activates the link $i, i=1,2$ maximizing

$$
\frac{\min \left(c_{i}(t), x_{i}(t)\right)}{\bar{c}_{i}} .
$$

Hence, the overall Joint stopping-rate allocation algorithm is given as follows. When mission $r$ arrives, start by $j=1$ :

1) if $u_{s}(j)>\mathbb{E}\left\{u_{s, m}(j+k)\right\}, \forall k=1 \ldots D-j$, stop

2) else, continue with

$$
r_{1}(t)=c_{1}(t), r_{2}(t)=0 \text { if } \frac{c_{1}(t)}{\bar{c}_{1}}>\frac{\min \left(c_{2}(t), x(t)\right)}{\bar{c}_{2}} . ;
$$

$$
\begin{aligned}
& r_{1}(t)=0, r_{2}(t)=\min \left(c_{2}(t), x(t)\right) \text { if } \frac{c_{1}(t)}{\bar{c}_{1}} \leq \\
& \frac{\min \left(c_{2}(t), x(t)\right)}{\bar{c}_{2}}
\end{aligned}
$$

The computations of $\mathbb{E}\left\{u_{s, m}(j+k)\right\}, \forall k=1 \ldots D-j$ involve evaluating expected utility values maximized over all combinations of link activations among the two hops for the next $k$ slots after stage $j$, given $u_{s}(j)$ for mission $s$. The algorithm can perform decisions with a low search space if any future stage with expected utility exceeding the current utility is readily found, i.e., it is not necessary to evaluate the optimizing configuration every stage.

We note that with a linear relationship assumption between $e(t)$ and $r(t)$, mean values of channel gains are sufficient to compute expected values for stopping decision. Even if these are not available apriori, the values may be learned over time.

We also point out that the equivalent Joint stopping-rate allocation algorithm for a single link involves only the first step, with only expectations evaluated for the next $k$ slots.

\section{Numerical Results}

We demonstrate the performance of the approximate algorithms via simulation results. First, we consider Fig. 3, which demonstrates the performance of different schedulers for dynamic links where each link of a two-hop relay network is ON or OFF with equal probability. The expiration deadline $D=20 s$, and $\gamma$, the timeliness parameter is varying. Delivered QoI utility increases with increasing $\gamma$, due to slower timeliness degradation. We observe that the approximate schedulers are near optimal for the immediate forwarding case, and significantly outperform schemes which stop equally likely at any time. Moreover, it is seen that the joint stopping-link activation algorithm provides significantly higher deliveredQoI utility with the aid of buffering compared with immediate forwarding. We also observe that schemes which possess knowledge of the channel state at the scheduling instant additionally improves the performance. Next, we present results for a tow-hop scenario with links subject to Rayleigh fading in Fig. 4, with $D=20 \mathrm{~s}$. We again observe that queues at relay nodes significantly improve delivered QoI utility.

Even with practical link schedulers, buffering demonstrates very significant improvements in QoI output utility compared with immediate forwarding. In fact, it is also seen that the hop-by-hop policy exceeds the maximum attainable utility (the OICC) achieved with immediate forwarding. Hence, similar to our results regarding stability regions [7], deploying queues at relay nodes also improves QoI utility via opportunistic scheduling. We also observe that the extent of improvement by hop-by-hop scheduling increases with greater variations in the channel states.

Finally, in Fig. 6 we demonstrate the effect of the number of hops on QoI utility. Scheduling is performed according to the adaptation of the joint stopping-rate allocation algorithm described in Section IV-B to more than two hops. While the attained utilities reduce due to increased delivery time for larger networks, we observe similar trends with the two-hop case. It is also worth noting that the relative performance gain due to buffering increases with number of hops. Flexibility in 


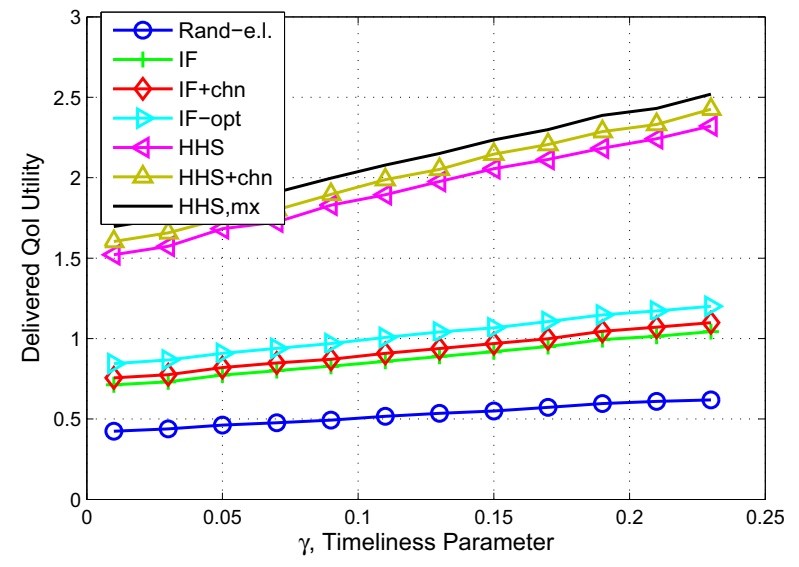

Fig. 4. QoI Utilities, ON-OFF links.

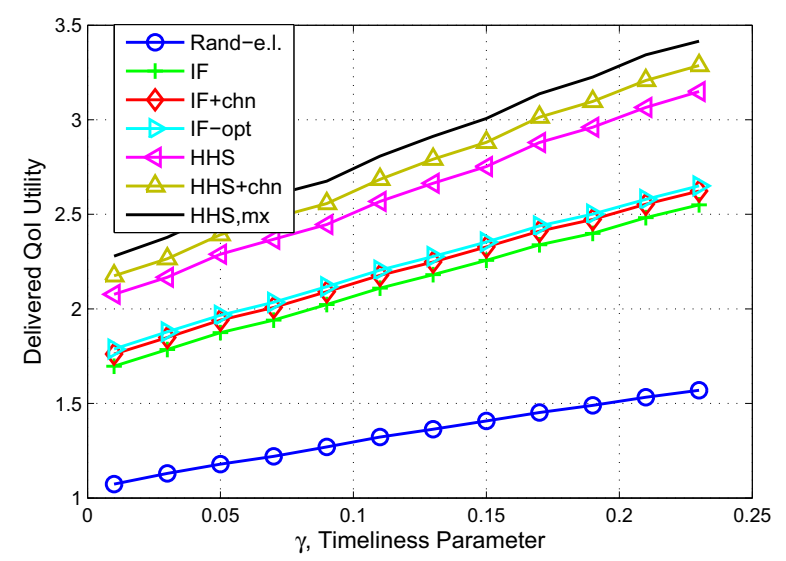

Fig. 5. QoI Utilities, Rayleigh Fading Links.

scheduling and avoiding hops with undesired channel realizations prevails over the effect due to the increased number of slots required to reach the final hop with buffering.

\section{CONCLUSIONS}

In this paper, we focus on the effect of network delivery and timeliness on information with specific input quality when the underlying channels are time varying. We first characterize the optimal solution for a single link, which is comparing the QoI utility with stage-dependent thresholds. Next, we propose joint QoI adaptation-link scheduling algorithms for multihop relay networks. We demonstrate that QoI utility delivered can be significantly increased by exploiting opportunistic scheduling via storage of information at relay nodes.

Storing data at relays and scheduling transmissions results in more flexibility to opportunistically exploit good channel states. We have also observed that the performance gap between the two approaches reduces with more balanced channel gain distributions, as immediate forwarding suffers less from inferior channel states due to diversity.

The approximate scheduling methods applied in this work can be readily generalized for multiuser scheduling in QoIbased networks with dynamic links. Future work includes extension of the policies to address cases where traffic arriving to the sources are stochastic.

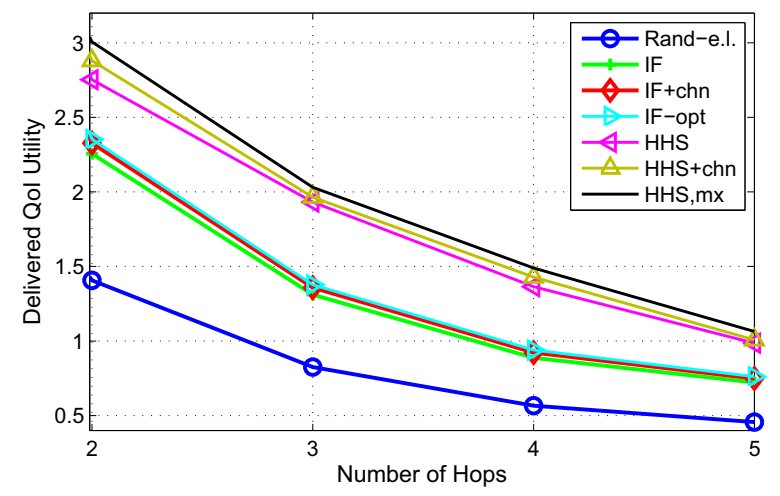

Fig. 6. QoI Utilities, $\gamma=0.15, \mathrm{D}=20$.

\section{REFERENCES}

[1] A. Bar-Noy, G. Cirincione, R. Govindan, S. Krishnamurthy, T. F. LaPorta, P. Mohapatra, M. Neely, and A. Yener. Quality-of-Information Aware Networking for Tactical Military Networks. In Third International Workshop on Information Quality and Quality of Service for Pervasive Computing, in conjunction with IEEE Percom 2011, Seattle, WA, March 2011.

[2] S. Zahedi, M. B. Srivastava, and C. Bisdikian. A Framework for QoI-inspired analysis for sensor network deployment planning. In International Workshop on Performance Control in Wireless Sensor Networks, PWSN, Austin, TX, October 2007.

[3] B. Liu, P. Terlecky, A. Bar-Noy, R. Govindan, and M. J. Neely. Optimizing Information Credibility in Social Swarming Applications. In IEEE INFOCOM 2011 mini-conference, Shanghai, China, April 2011.

[4] Z. M. Charbiwala, S. Zahedi Y. Kim, Y.H. Cho, and M. B. Srivastava. Toward Quality of Information Aware Rate Control for Sensor Networks. In Fourth International Workshop on Feedback Control Implemenation and Design in Computing Systems and Networks, San Francisco, CA, April 2009.

[5] R. Urgaonkar, E. N. Ciftcioglu, A. Yener, and M. J. Neely. Quality of Information Aware Scheduling in Task Processing Networks. In Proc. The 7th International Workshop on Resource Allocation and Cooperation in Wireless Networks (RAWNET), in conjunction with IEEE WiOpt 2011, Princeton, NJ, May 2011.

[6] E. N. Ciftcioglu, A. Yener, R. Govindan, and K. Psounis. Operational Information Content Sum Capacity: Formulation and Examples. In Proc. Fusion 2011, Chicago, IL, July 2011.

[7] E. N. Ciftcioglu, A. Yener, and R. A. Berry. Stability Regions of Bidirectional Cooperative Relaying. Submitted to IEEE Transactions on Information Theory, September 2010.

[8] T. M. Cover and J. A. Thomas. Elements of Information Theory. John Wiley and Sons, New York, NY, 1991.

[9] T. M. Cover and A. El Gamal. Capacity Theorems for the Relay Channel. IEEE Transactions on Information Theory, 25(5):572-584, September 1979.

[10] T. J. Oechtering and H. Boche. Stability Region of an Efficient Bidirectional Regenerative Half-duplex Relaying Protocol. In Proc. IEEE Information Theory Workshop, 2006. ITW '06, Chengdu, China, October 2006.

[11] T.J. Oechtering and H. Boche. Stability Region of an Optimized Bidirectional Regenerative Half-duplex Relaying Protocol. IEEE Transactions on Communications, 56(9):1519-1529, September 2008.

[12] D. Bertsekas. Dynamic programming and optimal control, vol.1. Athena Scienific, Bellmont, MA, 2001.

[13] T. Ferguson. Optimal Stopping and Applications. www.math.ucla.edu/tom/Stopping/Contents.html, 2006.

[14] L. Tassiulas and A. Ephremides. Stability Properties of Constrained Queuing Systems and Scheduling Properties for Maximum Throughput in Multihop Radio Networks. IEEE Transactions on Automatic Control, 37(12):1936-1948, December 1992.

[15] A. Jalali, R. Padovani, and R. Pankaj. Data throughput of CDMAHDR: a high efficiency-high data rate personal communication wireless system. In IEEE Vehicular Technology Conference Spring'00, Tokyo, Japan, May 2000. 